\title{
Towards understanding the molecular link between cell cycle regulation and hypoxic adaptation in Cryptococcus neoformans
}

Susumu Kawamoto ${ }^{1}$, Zuzana Moranova ${ }^{2}$, Eric V Virtudazo ${ }^{1}$, Misako Ohkusu ${ }^{1}$, Akiko Suganami $^{3}$, Yutaka Tamura ${ }^{3}$. Vladislav Raclavsky ${ }^{2}$

1. Medical Mycology Research Center, Chiba University, Chiba, Japan

2. Department of Microbiology, Faculty of Medicine and Dentistry, Palacky University, Olomouc, Czech Republic

3. Department of Bioinformatics, Graduate School of Medicine, Chiba University, Chiba, Japan

Objectives:

The objective of this study is to understand cell cycle regulation and hpoxic adaptation in the pathogenic yeast Cryptococcus neoformans and the molecular link between them.

Methods:

We performed in silico simulations and analyses using GENETYX software (version 15).

Results:

We have reported that the cell cycle behavior of the pathogenic yeast Cryptococcus neoformans (C. neoformans) is different from the cell cycle control exhibited by the model yeast

Saccharomyces cerevisiae (S. cerevisiae), and also have reported the molecular characterization and physiological roles of the two main eukaryotic cell cycle genes, $C$. neoformans cyclin

dependent kinase 1 (CnCdk1) and cyclin homologues. Only a single Cdk1-related G1 and G1/S cyclin homologue was found in the genome sequence of $C$. neoformans and was designated CnCln1. Surprisingly, $\mathrm{CnCln} 1$ was not only able to complement the function of the $\mathrm{G} 1$ cyclins of $S$. cerevisiae, such as $\mathrm{ScCln} 3$, but also the $\mathrm{G} 1 / \mathrm{S}$ cyclins of $S$. cerevisiae, such as $\mathrm{ScCln} 1$ and $\mathrm{ScCln} 2$. Our in silico analysis demonstrated that the $\mathrm{CnCln} 1 / \mathrm{ScCdk} 1$ complex was more stable than any of $S$. cerevisiae cyclins (ScCln1, ScCln2, ScCln3) and ScCdk1 complexes. These results are consistent with in vitro analysis that has revealed the flexible functional capacity of $\mathrm{CnCln} 1$ as a Cdk1-related G1 and $\mathrm{G} 1 / \mathrm{S}$ cyclin of S. cerevisiae. On the other hand, in S. cerevisiae, $\mathrm{Cln} 1$ and $\mathrm{Cln} 2, \mathrm{G} 1 / \mathrm{S}$ cyclins of $S$. cerevisiae, oscillate during the cell cycle, rising in late $\mathrm{G} 1$ and falling in early $\mathrm{S}$ phase. We have been trying to elucidate the structure basis of the functional distinction between $\mathrm{Cln} 1$ and $\mathrm{Cln} 2$.
We investigated the cell cycle control mechanism between $\mathrm{Cln} 1$ and $\mathrm{Cln} 2$ from a point of view of their structur

In the obligate aerobic pathogenic yeast $C$. neoformans, limited aeration has been demonstrated to cause slowdown in proliferation and delayed budding, resulting eventually in a unique unbudded G2-arrest. The ability to adapt to decreased oxygen levels during pathogenesis has been identified as a virulence factor in $C$. neoformans. We have identified and characterized the gene that is necessary for the proliferation slowdown and G2-arrest caused by limited aeration. This gene was also identified in a parallel studies as homologous both to calcineurin responsive (Crz1) and PKC1-dependent (SP1-like) transcription factors. We have confirmed the role of the cryptococcal homologue of CRZ1/SP1-like transcription factor in cell integrity, and newly

demonstrated its role in slowdown of proliferation and survival under reduced aeration, in biofilm formation and in susceptibility to fluconazole. Our data also demonstrate a tight molecular link between slowdown of proliferation during hypoxic adaptation and maintenance of cell integrity in C. neoformans and present a role for the CRZ1 family of transcription factors in fungi.

Our study revealed the flexible functional capacity of $\mathrm{CnCl} 1$ (only a single cyclin in C. neoformans) as a Cdk1-related G1 and G1/S cyclin (ScCln1, ScCln2, and ScCln3) of S. cerevisiae, and also demonstrate a tight molecular link between cell cycle regulation and hypoxic adaptation in $C$. neoformans.
(1)

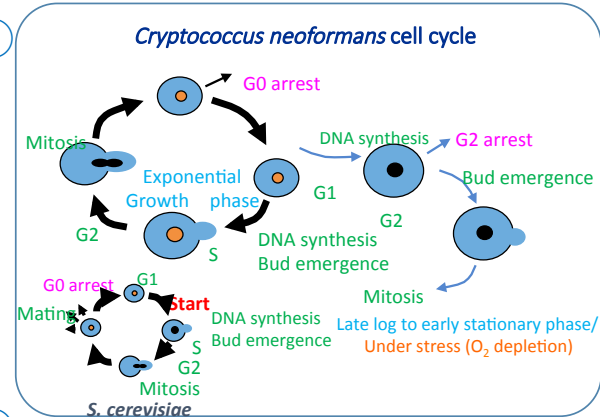

(4)
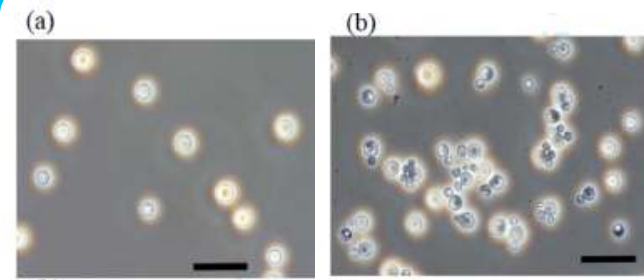

WT cells: Hypoxia-induced G2 arrest

Crz1/Sp1-deletd cells:

(6)

DELETING Crz1/Sp1 GENE REDUCES CELL NUMBER UNDER HYPOXIC CONDITION VERY SLIGHTLY

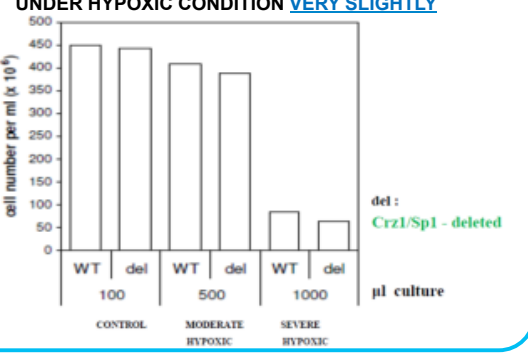

(9)

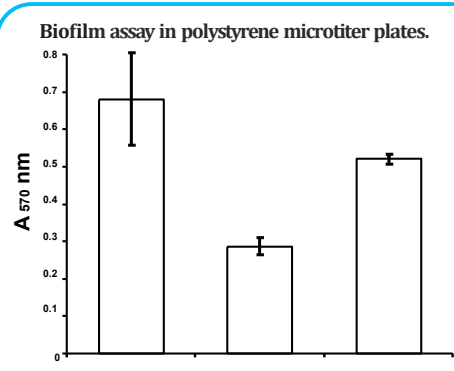

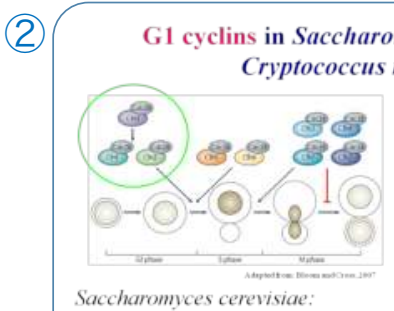

Saccharomyces cerevisia :

Evensive search of the completed $C$, neeformans genome database did not yield additional sequences with $\mathrm{G} 1$ cyclin similarities.

(5) Deleting Crz1/Sp1 Gene INCREASES CELL DEATH RATE UNDER HYPOXIC CONDITION REMARKABLY

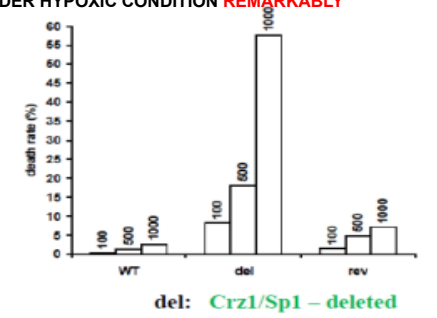

rev: reconstituted strain

(7) DELETING Crz1/Sp1 GENE REDUCES G2-FRACTION

UNDER HYPOXIC CONDITION REMARKABIY

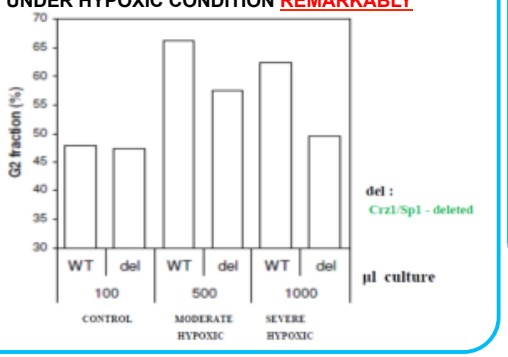

(10)
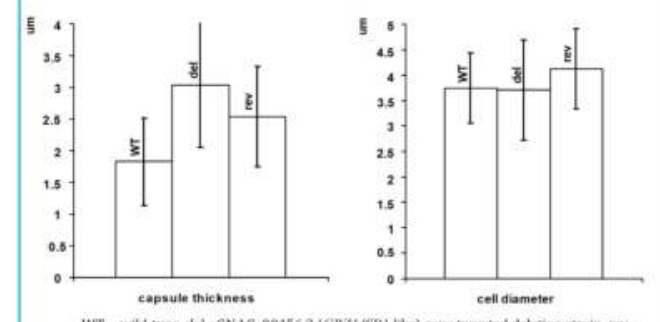

(3)

\begin{tabular}{lcc}
\hline & $\begin{array}{c}\text { Interaction Energy } \\
\text { (kestimal) }\end{array}$ & EC \\
\hline CnCin1/ ScCdk1 & -730.1 & 0.73 \\
SoCin1 / SoCdk1 & .730 .9 & 0.53 \\
SCCin2 / SoCok1 & -727.7 & 0.56 \\
SCCin3 / SCCok1 & -653.6 & 0.59 \\
\hline
\end{tabular}

(8)

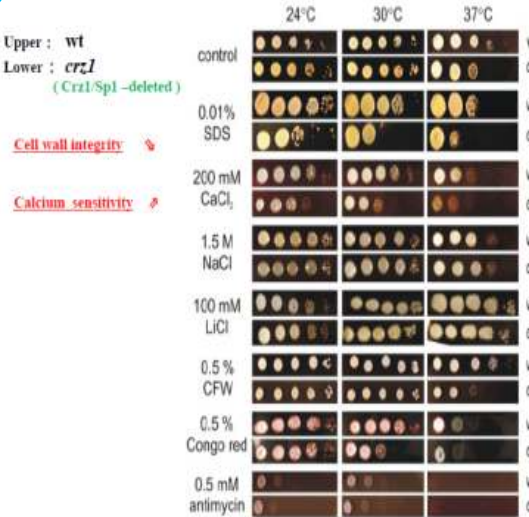

